\title{
A snapshot of extracellular DNA influence on Aspergillus biofilm
}

\author{
Luciana L. Guimarães ${ }^{1,2}$ and Helio K. Takahashi ${ }^{1 *}$ \\ ${ }^{1}$ Laboratory of Glycoconjugate Immunochemistry, Department of Biochemistry, Universidade Federal de São Paulo, São Paulo, Brazil \\ ${ }^{2}$ Laboratory of Natural Products, Department of Pharmaceutical Sciences, Universidade Santa Cecilia, Santos, Brazil \\ *Correspondence: takahashi.bioq@epm.br
}

Edited and reviewed by:

Maurizio Del Poeta, Stony Brook University, USA

Keywords: extracellular DNA, fungal biofilm, Aspergillus biofilm, extracellular matrix, antifungal resistance

\section{A commentary on}

Extrinsic extracellular DNA leads to biofilm formation and colocalizes with matrix polysaccharides in the human pathogenic fungus Aspergillus fumigatus by Shopova, I., Bruns, S., Thywissen, A., Kniemeyer, O., Brakhage, A. A., and Hillmann, F. (2013). Front. Microbiol. 4:141. doi: 10.3389/fmicb.2013.00141

\section{ASPERGILLOSIS IN CLINICAL CONTEXT}

Aspergillosis is a fungal infection that can assume a broad spectrum of clinical forms depending on immune status and the presence of underlying lung diseases, ranging from allergic bronchopulmonary aspergillosis to aspergilloma and also to the invasive aspergillosis forms that are a major cause of mortality in severely immunocompromised patients. Pulmonary aspergillosis follows the inhalation of airborne conidia of Aspergillus fumigatus, and pre-existing conditions such as pulmonary functional abnormalities such as cystic fibrosis or chronic obstructive pulmonary disease provides a favorable environment for Aspergillus colonization and biofilm formation in the lungs (Zmeili and Soubani, 2007; Ramage et al., 2011). Due to its great importance in clinical context, a better understanding of the process in filamentous growth and biofilm formation can help to manage Aspergillus infection, especially when dealing with resistant strains to current antifungal drugs (Arendrup, 2013).

\section{BIOFILMS AND THE RESISTANCE TO ANTIFUNGAL AGENTS}

Biofilms can be defined as microbial communities encased in a matrix of extracellular adhesive polymeric substances or extracellular matrix (ECM) and are considered as an important virulence determinant for a pathogen. For a fungus specie, growing as multicellular community may confer biological advantages in the colonization of tissues and may also act as barrier to external aggressions (Beauvais et al., 2007). Biofilms have raised special attention due to their high resistance to antimicrobial agents that can be up to 1000-fold greater than observed for planktonic cells (Tobudic et al., 2012). Previous studies with cultures of $A$. fumigatus maintained under static aerial conditions have demonstrated the presence of an ECM on the colony surface of colonial mycelia that acts as cohesive linkage bounding hyphae into a contiguous sheath, and this ECM is absent when the fungus is grown under liquid shake conditions. These colonies encased with ECM were also found to be extremely hydrophobic and displayed more resistance to antifungal polyenes amphotericin B and nystatin (Beauvais et al., 2007). In vivo studies showed that ECM production were also present at the surface of hyphae of A. fumigatus in an aspergilloma ("fungus ball") or during invasive aspergillosis (Loussert et al., 2010). In the antifungal resistance context, ECM may act as a physical barrier that decreases the access of antifungals to cells embedded in the biofilm community. The low penetration of the drugs seems to depend on the amount and nature of ECM, as well as the physicochemical properties of the antifungal agents. Thus, it was also demonstrated that the removal of ECM from Candida albicans biofilms reduced the survival rate of Candida cells when treated with Amphotericin B (Tobudic et al., 2012). The limited number of antifungal classes for treatment of aspergillosis brings special attention to biofilm formation in Aspergillus infections, considering the fact that the drugs should also be able to overcome the ECM barrier to perform its antifungal action.

\section{NATURE OF ASPERGILLUS BIOFILMS AND THE INFLUENCE OF eDNA}

Aspergillus ECM was initially found to be composed of galactomannan, alpha1,3 glucans, melanin and other proteins including hydrophobins (Beauvais et al., 2007). Recently it was demonstrated the presence of extracellular DNA (eDNA) in A. fumigatus ECM biofilm, which was released upon fungal autolysis (Rajendran et al., 2013). Taking into consideration that addition of DNAse improved in vitro activity of antifungal drugs directed to Candida albicans biofilms (Martins et al., 2012), it was suggested that the role of eDNA in Candida species may be associated with: (i) maintenance and stability of biofilms and (ii) biofilm resistance to antifungal drugs. Similar results were also observed for A. fumigatus biofilms, where the treatment with DNAse destabilized biofilm architecture integrity and concomitant treatment of biofilms with DNAse and amphotericin B or caspofungin improved antifungal susceptibility (Rajendran et al., 2013). These findings together demonstrated the important role of eDNA in Aspergillus ECM biofilm. Further studies exploiting the role of eDNA in biofilm formation performed by Brakhage and Hillman's research group, have demonstrated the importance of extrinsic eDNA in inducing and shaping $A$. fumigatus biofilms by adding exogenous eDNA in an in vitro biofilm model (Shopova et al., 2013). They also showed that eDNA 
facilitated surface adhesion of fungal spores and also colocalized with ECM biofilm polysaccharides, becoming part of the ECM surrounding the biofilm cells. The fact that exogenous eDNA stimulated biofilm formation in vitro resembles body's innate immune response to inhaled conidia, whereas the response to conidia that manage to escape alveolar macrophages includes the formation of neutrophil extracellular traps (NETs) which consist in masses of decondensed chromatin decorated with antimicrobial proteins and opsonins (McCormick et al., 2010). Within this context, NETs are believed to function as physical barrier containing the microorganism within the mass of chromatin, enhancing direct contact with the antimicrobial NETsassociated proteins, thus representing an anti-microbial effector mechanism that mediates killing of a wide range of bacterial pathogens as well as C. albicans (McCormick et al., 2010). However, in vitro models with Aspergillus showed that despite the fact that NETs formation leads to trapping the fungus conferring a fungistatic effect by containing the infection, it does not represent the major factor for killing this fungus since NET-dependent killing of Aspergillus hyphae was only moderated (Bruns et al., 2010).

\section{FUTURE PERSPECTIVES}

Taken together these findings, the next steps would include the elucidation of the in vivo influence of NET's eDNA produced by lungs in Aspergillus biofilm development, which may lead to the combination of antifungal drugs with DNAse to manage pulmonary aspergillosis. Further studies could also explore the Aspergillus ECM physicochemical features and permeability to current antifungal drugs, and these findings may lead to molecular improvement of these drugs by conferring them the capacity to penetrate ECM Aspergillus biofilm and act in their respective molecular targets.

\section{ACKNOWLEDGMENT}

The authors would like to acknowledge Prof. Anita H. Straus for critically reviewing the manuscript.

\section{REFERENCES}

Arendrup, M. C. (2013). Update on antifungal resistance in Aspergillus and Candida. Clin. Microbiol. Infect. doi: 10.1111/1469-0691.12513. [Epub ahead of print].

Beauvais, A., Schmidt, C., Guadagnini, S., Roux, P., Perret, E., Henry, C., et al. (2007). An extracellular matrix glues together the aerialgrown hyphae of Aspergillus fumigatus. Cell. Microbiol. 9, 1588-1600. doi: 10.1111/j.14625822.2007.00895.x

Bruns, S., Kniemeyer, O., Hasenberg, M., Aimanianda V., Nietzsche, S., Thywissen, A., et al. (2010). Production of extracellular traps against Aspergillus fumigatus in vitro and in infected lung tissue is dependent on invading neutrophils and influenced by hydrophobinroda. PLoS Pathog. 6:e1000873. doi: 10.1371/journal.ppat.10 00873

Loussert, C., Schmitt, C., Prevost, M. C., Balloy, V., Fadel, E., Philippe, B., et al. (2010). In vivo biofilm composition of Aspergillus fumigatus. Cell. Microbiol. 12, 405-410. doi: 10.1111/j.14625822.2009.01409.x

Martins, M., Henriques, M., Lopez-Ribot, J. L., and Oliveira, R. (2012). Addition of DNase improves the in vitro activity of antifungal drugs against Candida albicans biofilms. Mycoses 55, 80-85. doi: 10.1111/j.1439-0507.2011. 02047.x

McCormick, A., Loeffler, J., and Ebel, F. (2010). Aspergillus fumigatus: contours of an opportunistic human pathogen. Cell. Microbiol. 12, 1535-1543. doi: 10.1111/j.1462-5822.2010.01517.x

Rajendran, R., Williams, C., Lappin, D. F., Millington, O., Martins, M., and Ramage, G. (2013). Extracellular DNA release acts as an antifungal resistance mechanism in mature Aspergillus fumigatus biofilms. Eukaryot. Cell 12, 420-429. doi: 10.1128/EC.00287-12

Ramage, G., Rajendran, R., Gutierrez-Correa, M., Jones, B., and Williams, C. (2011). Aspergillus biofilms: clinical and industrial significance. FEMS Microbiol. Lett. 324, 89-97. doi: 10.1111/j.15746968.2011.02381.x

Shopova, I., Bruns, S., Thywissen, A., Kniemeyer, O., Brakhage, A. A., and Hillmann, F. (2013). Extrinsic extracellular DNA leads to biofilm formation and colocalizes with matrix polysaccharides in the human pathogenic fungus Aspergillus fumigatus. Front. Microbiol. 4:141. doi: 10.3389/fmicb.2013.00141

Tobudic, S., Kratzer, C., Lassnigg, A., and Presterl, E. (2012). Antifungal susceptibility of Candida albicans in biofilms. Mycoses 55, 199-204. doi: 10.1111/j.1439-0507.2011.02076.x

Zmeili, O. S., and Soubani, A. O. (2007). Pulmonary aspergillosis: a clinical update. Q. J. Med. 100, 317-334. doi: 10.1093/qjmed/hcm035

Conflict of Interest Statement: The authors declare that the research was conducted in the absence of any commercial or financial relationships that could be construed as a potential conflict of interest.

Received: 27 April 2014; accepted: 12 May 2014; published online: 30 May 2014.

Citation: Guimarães LL and Takahashi HK (2014) A snapshot of extracellular DNA influence on Aspergillus biofilm. Front. Microbiol. 5:260. doi: 10.3389/fmicb. 2014.00260

This article was submitted to the journal Frontiers in Microbiology.

Copyright (c) 2014 Guimarães and Takahashi. This is an open-access article distributed under the terms of the Creative Commons Attribution License (CC BY). The use, distribution or reproduction in other forums is permitted, provided the original author(s) or licensor are credited and that the original publication in this journal is cited, in accordance with accepted academic practice. No use, distribution or reproduction is permitted which does not comply with these terms. 